\title{
Alianza Tríadica Familiar y Salud Mental Parental
}

\section{Triadic Family Alliance and Parental Mental Health}

\author{
Francisca Pérez C \\ Facultad de Psicología, Universidad Alberto Hurtado, Chile. \\ María Pía Santelices A. \\ Escuela de Psicología, Pontificia Universidad Católica de Chile, Chile.
}

Rec (25 de junio de 2015) Acept (24 de febrero 2017)

\begin{abstract}
Resumen
El Lausanne Trialogue Play (LTP), instrumento que permite la evaluación estandarizada de la alianza familiar, releva la importancia del padre en los estudios de procesos e interacciones familiares, así como la existencia de un efecto familiar tríadico desde etapas tempranas. El presente estudio pretende aportar al conocimiento de la alianza familiar en familias con hijos(as) en edad preescolar, analizando su relación con la salud mental de los padres. 50 parejas parentales y sus hijos(as) fueron evaluadas en sus hogares con una batería de instrumentos que incluían el LTP, BDI-I, PSI-SF y un Cuestionario Sociodemográfico. Los resultados muestran que la mayoría de las familias evaluadas presentan alianzas familiares disfuncionales, sin evidenciarse una relación estadísticamente significativa con las variables de salud mental estudiadas. Sin embargo, se observa una tendencia a que familias con alianzas conflictivas presenten mayores niveles de sintomatología depresiva y estrés parental, sobre todo en el rol paterno.

Palabras clave: alianza familiar, sintomatología depresiva, estrés parental.
\end{abstract}

\begin{abstract}
The Lausanne Trialogue Play (LTP) is an instrument that allows the standardized assessment of the family alliance, highlighting the importance of the father in studies of family process and interactions, as also the existence of a triadic family effect since early stages. The present study pretends to contribute to extend the knowledge in family alliances in families with preschool aged children, analyzing it's relation with the parental mental health variables. 50 parental couples and their children were evaluated at their homes with the following instruments: LTP, BDI-I, PSI-SF and a sociodemographic questionnaire. Results show that most of the families have dysfunctional alliances, without proving a statistically significant relation with the parental studied variables. Nevertheless, families with conflictive alliances tend to show higher levels of depressive symptoms and parental stress, especially in fathers. Key words: family alliance, depressive symptoms, parental stress.
\end{abstract}

\footnotetext{
Correspondencia: Francisca Pérez C., Facultad de Psicología, Universidad Alberto Hurtado, Santiago, Chile. Almirante Barroso 10, Santiago de Chile. Teléfono: 56228897436. E-mail: frperez@uahurtado.cl

Este artículo contó con el apoyo financiero de Fondo Nacional de Desarrollo de la Ciencia y Tecnología, FONDECYT, No 1100721 y al Fondo de Innovación para la Competividad (FIC) del Ministerio de Economía, Fomento y Turismo, a través de la Iniciativa Científica Milenio, Proyecto IS130005.
} 


\section{Introducción}

\section{Más allá de la díada}

El núcleo familiar es el sistema que define y configura en mayor medida el desarrollo de la persona desde su concepción (Bronfenbrenner, 1987), de manera que las relaciones recíprocas entre sus integrantes son centrales en la emergencia de un estilo familiar armónico y cohesivo que apoye un desarrollo óptimo sus miembros (Belsky, 1981; Cowan y Cowan, 2002; Fincham, 1998; McHale y Cowan, 1996).

Si bien históricamente las investigaciones en procesos e interacciones familiares se han centrado en la díada madrehijo(a) (Flykt, Kanninen, Sinkkone, y Punamaki, 2010; Teti, Gelfand, Messinger, y Isabella, 1995; Weinberg y Tronick, 1998), los primeros estudios que incluyeron al padre mostraron que su presencia tiene un impacto en las conductas de la madre y del hijo(a) y en la calidad del intercambio emocional entre ambos; influencia que sugiere un efecto familiar tríadico (Clarke-Stewart 1978; Yogman 1981).

La teoría sistémica familiar y la psicología del desarrollo se han combinado para ir más allá de la díada "madrehijo(a)" a la tríada "madre-padre-hijo(a)", postulando que el niño(a), desde el momento de su nacimiento, está expuesto e inserto en contextos relacionales más amplios que estrictamente diádicos, y que a partir de los tres o cuatro meses de edad es capaz de distribuir su atención entre ambos padres, de manera que las relaciones triangulares se vuelven parte importante en su desarrollo (Dunn, 1991; Fivaz-Depeursinge, Corboz-Warnery, Keren, 2004).

El Lausanne Trialogue Play (LTP) desarrollado por Fivaz-Depeursinge, Frascarolo y Corboz-Warnery (1996) constituye un aporte en esta dirección, ya que es una herramienta que a través del análisis de los distintos subsistemas de la tríada y cómo estos se organizan en un todo adaptativo (Cox y Paley, 1997), permite examinar la interacción familiar, ampliando así la parcialidad de las evaluaciones díadicas.

El presente estudio pretende dar cuenta de la alianza familiar, a través del análisis del LTP, en familias con niños(as) en edad preescolar, ya que durante este periodo el juego constituye una forma natural de relacionarse. Dado que en esta etapa el desarrollo de las habilidades motoras, sensoriales y verbales del niño(a) posibilitan el surgimiento de la teoría de la mente, es decir, la capacidad de representarse el mundo, a las personas que los rodean y a sí mismos, el niño(a) ya es capaz de relacionarse con otros en un discurso emocional y representacional (Ontai, y Thompson, 2002), lo que expresará en el juego. Además a esta edad, el ingreso al jardín infantil pone a prueba la capacidad de adaptación del niño(a) y por ende de todo el sistema familiar, de manera que muchas veces es en esta etapa cuando se detectan por primera vez algunos desajustes o problemas en el funcionamiento familiar.

Existen múltiples factores psicosociales de riesgo que pueden tener consecuencias negativas en la capacidad familiar de mantener interacciones de calidad (Gómez, Muñoz, y Haz, 2007; Vick Whittaker, Jones Harden, See, Meisch, Westbrook, 2011), siendo la salud mental de los padres uno de estos. Es así como la presente investigación pretende aportar al conocimiento de la relación entre interacciones familiares tríadicas "madre-padre-hijo(a)" y salud mental parental.

\section{Alianzas tríadicas}

Mientras que en una díada hay solo una forma posible de interacción, la investigación del juego tríadico resulta más compleja, ya que es necesario explorar cuatro tipos de configuraciones (tres díadas, una tríada), dado que cada integrante puede asumir un rol activo o tomar una posición pasiva. Esta dificultad puede enfrentarse distinguiendo cuatro funciones que se dan en las interacciones, las cuales se encuentran imbricadas y siguen un orden jerárquico: 1) la participación que considera la inclusión de todos los participantes, 2) la organización que implica que los integrantes se mantengan en su rol, 3) la focalización, es decir, que se comparta un foco común de atención y 4) y el contacto afectivo donde todos los miembros estén conectados. El análisis de estas funciones en las configuraciones familiares a través del juego permite identificar la funcionalidad o disfuncionalidad de las interacciones familiares "madre-padre-hijo(a)", las cuales se pueden categorizar en "alianzas tríadicas" o "alianzas familiares" de acuerdo al grado de coordinación logrado por sus integrantes (Fivaz-Depeursinge, y CorbozWarnery, 1999).

Dado que un objetivo natural de la tríada es crear sentimientos lúdicos y cooperación entre sus participantes, el logro de una interacción exitosa implica tanto la capacidad de coordinación familiar, así como la capacidad de reorganizar la interacción después de una pausa o variación en el tema de intercambio y de reparar las descoordinaciones sin interrumpir el intercambio en curso (Fivaz-Depeursinge y Corboz-Warnery, 1999; Frascarolo, Favez, Carneiro, FivazDepeursinge, 2004; Stern, 1977).

Cuando la tríada logra una interacción de calidad, se postula que presenta una "alianza cooperativa", la cual 
describe a familias cuyos miembros participan, logran coordinarse "suficientemente bien" en torno a una tarea conjunta, respetan sus roles e intercambian afectos positivos. El evaluador tiene la impresión que las interacciones son coherentes y cohesionadas. La "alianza conflictiva", por su parte, describe a familias cuyos miembros compiten, lo que se expresa a través de un conflicto que puede ser abierto o encubierto. Los padres no son capaces de coordinarse "suficientemente bien" para llevar a cabo la tarea, interrumpiéndose, provocando quiebres en la interacción y compitiendo por obtener la atención del niño(a). Si bien logran realizar juegos diádicos, les resulta más difícil realizar juegos tríadicos. Por último, la "alianza desorganizada" describe a familias que muestran interacciones que se caracterizan por la exclusión de uno de sus miembros y que a pesar de sus esfuerzos, no logran coordinación, cohesión ni contacto emocional entre sí.

Si bien, son múltiples los factores que pueden incidir en la funcionalidad o disfuncionalidad de la alianza familiar, son aquellos aspectos más cercanos al individuo los que parecen incidir de manera más directa, de manera tal que la salud mental de los padres, resulta un factor relevante a considerar en la evaluación de las interacciones familiares (Pérez, Lorence y Menéndez, 2010).

\section{Salud mental parental e interacciones familiares}

Ha sido ampliamente reportado en investigaciones que la presencia de sintomatología depresiva en las madres disminuye sus niveles de reciprocidad, sincronía y coordinación con su hijo(a), comprometiéndose la capacidad diádica y tríadica que permite la regulación afectiva (Feldman, 2007). Asimismo madres que presentan altos niveles de estrés refieren sentirse sobrepasadas frente a las demandas de sus hijos(as), estando menos disponibles para responder a sus necesidades (Rodrigo, Martín, Cabrera y Máiquez, 2009).

Si bien el padre ha sido significativamente menos estudiado que la madre, durante las últimas tres décadas han proliferado los estudios que lo incorporan (Cabrera, Tamis-LeMonda, Bradley, Hofferth y Lamb, 2000); Lamb, 2004) revisar esta cita no se encuentra en las referencias AGREGADA; Tenorio, Santelices y Pérez, 2009). Algunos de estos han mostrado que su presencia tiene un impacto en las conductas de cada miembro de la díada madre-hijo(a) y en la calidad del intercambio emocional.

En relación con la presencia e involucramiento del padre, algunos estudios han planteado que esta varía de acuerdo a la relación que tenga el padre con el niño(a), su capital humano, su bienestar psicológico y fuentes de soporte social y estrés (Belsky, 1984; Hossain y Roopnarine, 1994; Pleck y Masciadrelli, 2004). Por ejemplo, padres con mayor nivel educacional son más capaces de proveer a sus hijos e involucrarse con ellos (McLanahan, 2009), mientras que padres con mayor sintomatología depresiva y estrés muestran mayores niveles de hostilidad, rechazo y estimulación inadecuada (Cummings et al., 2000; Parke et al, 2004). Otros estudios señalan que la sintomatología depresiva paterna varía de acuerdo a su estado civil y ocupación y que se asocia negativamente con la relación padre-hijo(a), padre-madre y positivamente con el estrés parental (Bronte-Tinkew, Moore, Matthews, y Carrano, 2007).

Las investigaciones en salud mental infantil y familiar muestran que la participación del niño(a) en la tríada que integra junto a su madre y su padre, puede ayudar a resolver interacciones diádicas disfuncionales con uno de ellos. Dickstein et al. (1998) plantean que un mediador significativo del desarrollo de niños(as) preescolares expuestos a psicopatología materna es la salud del funcionamiento familiar. En familias en las cuales uno de los padres posee una enfermedad mental, el desarrollo del niño(a) depende más del nivel de funcionamiento familiar que de la interacción diádica que mantenga con el padre (o la madre) que presente una patología (Seifer y Dickstein, 2000). Esta experiencia favorece además la regulación emocional en el niño(a) durante la interacción, contribuyendo a la reducción de la tensión y el estrés (Fivaz-Depeursinge y Favez, 2006). Por su parte, existe alguna evidencia que indicaría que los padres son sensibles a los riesgos maternos, incluyendo sintomatología depresiva (Cummings, Goeke-Morey, y Raymond, 2004) y que la adecuada salud mental del padre actúa como un factor protector para el niño(a) en la relación entre la sintomatología depresiva materna e infantil (Gere, Hagen, Villabo, Arnberg, Neumer y Torgersen, 2013).

En el uso del LTP en contextos no clínicos se ha identificado que los bebés interactúan con ambos padres intercambiando a través de la mirada señales de placer $o$ displacer de forma equitativa (Lavanchy, 2002). En familias con alianzas funcionales estos intercambios son más frecuentes que en familias con alianzas disfuncionales. Asimismo el intercambio de afectos placenteros por sobre afectos displacenteros suele ser más frecuente en familias con alianzas funcionales en comparación con familias disfuncionales (Koller, 2004). En otras palabras, en familias con alianzas problemáticas los intercambios emocionales entre el niño(a) y sus padres suelen ser más negativos. Por su parte, el uso del LTP en contextos clínicos ha mostrado 
que la capacidad tríadica es significativamente más baja en familias con antecedentes clínicos (Gertsch Bettens, Favez, Corboz-Warnery y Fivaz-Depeursinge, 1992).

A partir de los antecedentes expuestos, la presente investigación pretende aportar al estudio de la relación entre alianza tríadica y salud mental parental. Se espera que las tríadas compuestas por padres que presentan mayor sintomatología clínica presenten alianzas familiares más disfuncionales, mientras que familias en las cuales ambos padres presenten bajos niveles de sintomatología depresiva y estrés se observen alianzas cooperativas. Asimismo se indagará esta relación en parejas en las cuales madres y padres presenten distintos niveles de sintomatología depresiva y estrés parental. Adicionalmente, se analizará la relación entre salud mental y capital cultural (nivel educacional, ocupación) de los padres.

\section{Método}

Diseño

El presente estudio es transversal y de carácter exploratorio, ya que se trata de un estudio pionero con LTP en familias con niños/as de edad preescolar en el medio nacional.

\section{Participantes}

Participaron 50 tríadas (poder y confiabilidad 95\%), específicamente madres, padres y sus hijos(as) residentes en distintas comunas de la ciudad de Santiago. Las madres tenían una edad promedio de 32,2 años (D.S=6,2), los padres tenían en promedio 33,4 años (D.S=6,7) y los niños(as) tenían en promedio 4,5 años (D.S.=2,7). El nivel educacional y ocupación de los padres se puede apreciar en la tabla $\mathrm{N}^{\circ} 1$. Es importante mencionar que en el $80 \%$ de las familias las madres eran definidas como cuidadores principales, en el $4 \%$ los padres, $4 \%$ abuelos, $2 \%$ tíos y en un $10 \%$ otros.

Los criterios de inclusión para las familias participantes era vivir en pareja y tener al menos un hijo(a) de edad preescolar que asistiera de forma permanente al jardín infantil. Los criterios de exclusión era padecer alguna enfermedad física inhabilitante o algún trastorno psiquiátrico diagnosticado.

\section{Procedimiento}

Se seleccionaron jardines infantiles públicos y gratuitos que estuvieran ubicados en la ciudad de Santiago de los cuales se eligieron 24 de forma aleatoria. Una vez establecidos los contactos con los jardines, se procedió a seleccionar a las familias que contaran con los criterios de inclusión. Las familias seleccionadas fueron contactadas telefónicamente, instancia en la cual se las invitaba a participar libremente y si accedían se agendaba una visita domiciliaria en la cual se llevaba a cabo la evaluación. Dos evaluadoras con entrenamiento previo en los instrumentos asistían a los hogares, y en 1,5 horas llevaban a cabo la evaluación completa, que consistía en la firma del consentimiento informado por ambos padres, la aplicación de los cuestionarios y la grabación del juego tríadico.

Tabla 1. Descripción de las variables sociodemográficas.

\begin{tabular}{|c|c|c|c|c|}
\hline Variable & $\begin{array}{c}\text { Frecuencia } \\
\text { Madres }\end{array}$ & $\begin{array}{c}\text { Porcentaje } \\
\text { Madres }\end{array}$ & $\begin{array}{c}\text { Frecuencia } \\
\text { Padres }\end{array}$ & $\begin{array}{c}\text { Porcentaje } \\
\text { Padres }\end{array}$ \\
\hline \multicolumn{5}{|l|}{ Escolaridad: } \\
\hline -Básica incompleta & 2 & $4 \%$ & 2 & $4 \%$ \\
\hline -Básica completa & 5 & $10 \%$ & 4 & $8 \%$ \\
\hline -Media incompleta & 9 & $18 \%$ & 16 & $32 \%$ \\
\hline -Media completa/ técnica incompleta & 25 & $50 \%$ & 21 & $42 \%$ \\
\hline -Técnica completa/uni incompleta & 8 & $16 \%$ & 7 & $14 \%$ \\
\hline -Universitaria completa & 1 & $2 \%$ & & \\
\hline \multicolumn{5}{|l|}{ Ocupación: } \\
\hline -Dueñas de casa /cesante & 28 & $56 \%$ & 5 & $10 \%$ \\
\hline -Trabajos menores e informales & 1 & $2 \%$ & 1 & $2 \%$ \\
\hline $\begin{array}{l}\text {-Oficio menor, obrero no calificado, servicio doméstico } \\
\text { con contrato }\end{array}$ & 7 & $14 \%$ & 15 & $30 \%$ \\
\hline -Obrero calificado, junior, micro empresario & 9 & $18 \%$ & 23 & $46 \%$ \\
\hline $\begin{array}{l}\text {-Empleado administrativo, vendedor, secretaria, técnica } \\
\text { especializada }\end{array}$ & 5 & $10 \%$ & 6 & $12 \%$ \\
\hline
\end{tabular}


Instrumentos

\section{Características sociodemográficas familiares}

Se creó un cuestionario específico que evaluaba antecedentes del desarrollo del niño(a), así como antecedentes sociodemográficos de la familia (constitución familiar, nivel educacional, ocupación, etc.).

\section{Inventario de Depresión de Beck (BDI-I)}

Instrumento desarrollado por Beck, Ward, Mendelson, Mock y Erlbaugh (1961), con el fin de evaluar la presencia de sintomatología depresiva en el adulto. Es un cuestionario auto-aplicado, que constan de 21 preguntas cerradas, que describen diversos síntomas de depresión. Estas se evalúan de acuerdo a una escala Likert de 4 categorías, puntuándose cada una de ellas entre 0 a 3 puntos. La puntuación máxima posible es 63 puntos, donde un mayor puntaje indica mayor presencia de sintomatología. El instrumento ha sido ampliamente usado en diversos estudios, demostrando buenos índices de confiabilidad y validez (0.92) (Beck et al, 1996 citado en Melipillán, Cova, Rincón y Valdivia, 2008).

\section{Inventario de estrés parental formato abreviado (PSI-SF)}

Instrumento desarrollado por Abidin (1995), que consiste en 36 ítems derivados del instrumento original PSI y se compone de tres escalas: estrés parental, características del niño(a) difícil e interacciones parento-filiales disfuncionales. Los puntajes de corte para la escala general son los siguientes: 0-20 indican niveles leves de estrés; 21-84 niveles medios de estrés; 85 o más puntos indican altos niveles de estrés (Abidin, 1995).

El uso de su forma abreviada se eligió considerando limitaciones temporales y dado que los niveles de consistencia interna del instrumento general y por subescalas es similar al instrumento original (Roggman, Moe, Hart y Forthun, 1994) con coeficientes entre 0,78 y 0,90 en una muestra con 103 padres. Estudios de confiabilidad realizados por Abidin (1995) en una muestra de 800 casos muestran buenos coeficientes test-retest $(0,68-0,85)$ y consistencia interna (0,80-0,90). Un estudio chileno llevado a cabo con 137 madres de estrato socioeconómico bajo estableció un alpha de Cronbach de .94 (Farkas, 2011). La validez externa fue establecida con la escala completa obteniéndose coeficientes de correlación entre 0,73 y 0,95 (Abidin, 1995).

\section{Lausanne Trialogue Play (LTP) versión preescolar}

Instrumento observacional desarrollado por FivazDepeursinge, Frascarolo y Corboz-Warner (1996) con el fin de evaluar de forma estandarizada la interacción entre madre-padre e hijo. La familia se sienta alrededor de una mesa, de manera tal que entre los tres integrantes tengan contacto visual. A través de una consigna la familia es alentada a jugar en cuatro fases: 1) padre o madre juegan activamente con el niño mientras el otro adulto solo está presente, 2) luego estos roles se invierten entre los padres, 3) luego padre, madre y niño juegan activamente y 4) finalmente padre y madre interactúan y el niño simplemente está presente. Las interacciones se filman con dos cámaras sincronizadas, una enfocando a los padres y la otra al niño (figura 1).

El procedimiento permite determinar tres tipos de alianza familiar con dos opciones en cada una de ellas: 1) Alianza cooperativa, la que puede ser a) fluida o b) tensa, 2) Alianza conflictiva, la que puede ser a) encubierta o b) abierta y Alianza desorganizada, la que puede ser a) con exclusión o b) caótica. El tipo de alianza familiar se determina a partir de la evaluación de las funciones estructurales y dinámicas de la interacción, las cuales se clasifican en niveles adecuados, moderados o inadecuados.

Cabe mencionar que en el siguiente estudio los videos fueron codificados por dos jueces expertos obteniéndose buenos de confiabilidad inter-evaluador (kappa=0,7, $\mathrm{p}<0,05$ ).

\section{Análisis de datos}

Se llevaron a cabo análisis descriptivos de las alianzas familiares, las variables de salud mental de los padres y las variables sociodemográficas familiares. Además se calculó la confiabilidad inter-evaluador de las alianzas a través del índice kappa. Con fines de comparar los promedios de la sintomatología depresiva y estrés en los padres según el tipo de alianza familiar se llevaron a cabo análisis de pruebas $\mathrm{t}$ de Student y de varianza. Para cada uno de los análisis se calculó el tamaño del efecto de Cohen, considerando que menor a 0,2 corresponde a un tamaño del efecto pequeño, 0,5 mediano y 0,8 grande. Por último para establecer la relación entre la salud mental de los padres y las características sociodemográficas de las familias se efectuaron análisis correlacionales de tipo bivariado. 


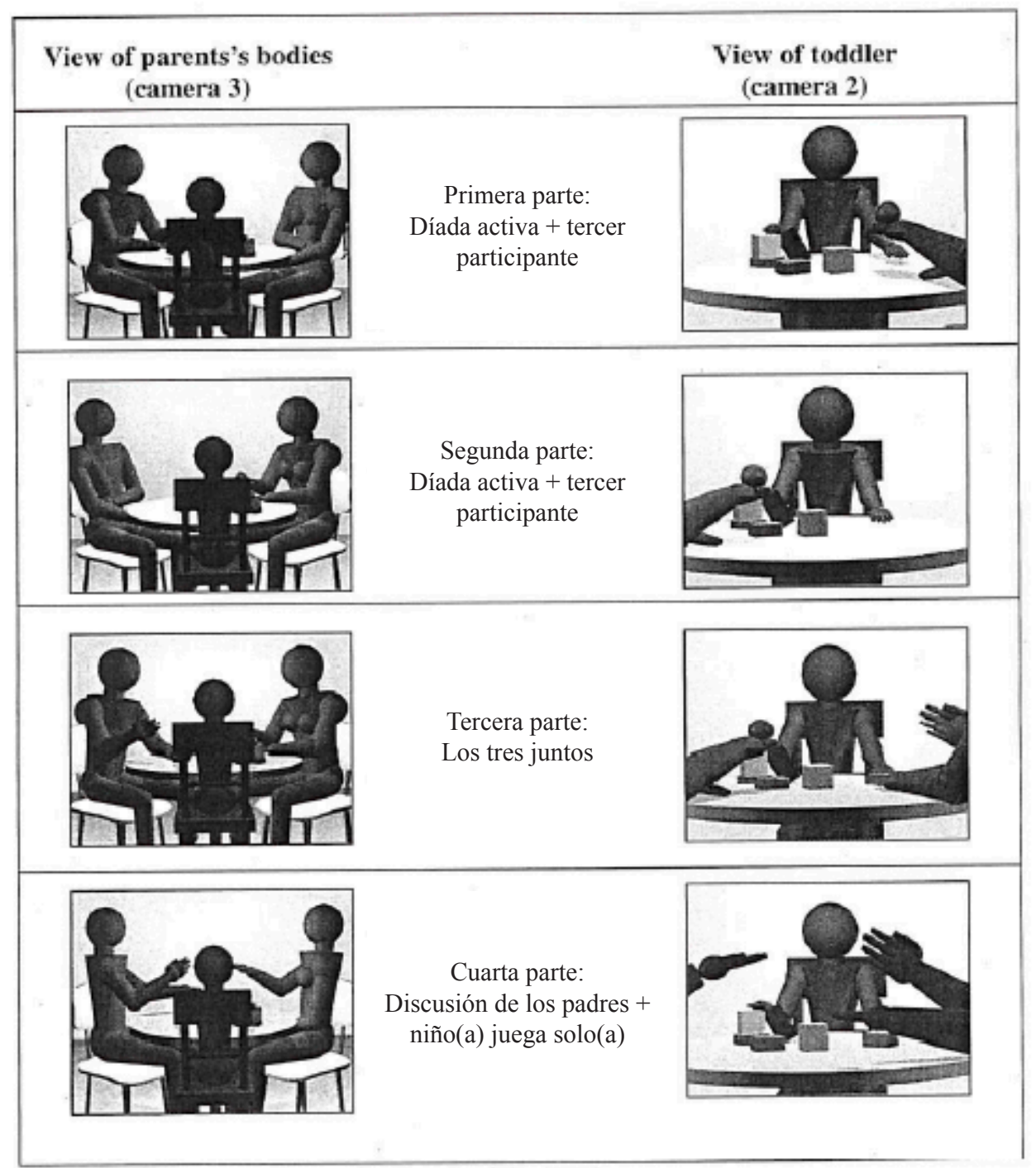

Nota. Fuente. Centre d'Etude de la Famille (2007). Figure 2: Views according to the 4 parts of the LTP. Indications for the LTP Setting for Toddlers. VB/ 15.02 .98 ; translation : CLS y FF/2007.

Figura 1. Vista de las cuatro posiciones del LTP

\section{Resultados}

\section{Análisis descriptivos}

De las familias evaluadas un $10 \%$ presentaron alianzas cooperativas, donde solo una mostró el subtipo fluido y cuatro familias (8\%) el subtipo tensa. Un 70\% de las familias presentaron alianzas de tipo conflictiva, donde un $66 \%$ presentó del tipo encubierto y un $4 \%$ del tipo abierto. Y por último, un $20 \%$ presentó alianzas desorganizadas, donde un $12 \%$ de estas eran con exclusión y un $8 \%$ de tipo caótica.

De acuerdo a los resultados obtenidos en el BDI-I (ver tabla $\mathrm{N}^{\circ}$ ) madres y padres puntúan en promedio niveles mínimos de sintomatología depresiva y no existen diferencias significativas entre ambos grupos $(\mathrm{t}=1.6, \mathrm{p}>.05)$. Un $74 \%$ de las madres presentó sintomatología mínima, un 18\% sintomatología leve y un $8 \%$ sintomatología moderada, mientras que los padres un $78 \%$ sintomatología mínima, un 16\% sintomatología leve, un $2 \%$ moderada y un $4 \%$ sintomatología severa.

En relación con los puntajes obtenidos en el PSI-SF todas las sub-escalas se encuentran en los niveles medios de estrés y solo se observan diferencias significativas en la sub-escala estrés parental $(\mathrm{t}=2.49, \mathrm{p}<.05)$ entre madres $\mathrm{y}$ padres, donde las madres muestran mayores niveles. Un $60 \%$ de las madres presenta niveles medios de estrés y un $40 \%$ niveles altos, mientras que un $69,4 \%$ de los padres presenta niveles medios y un 30,6\% niveles altos de estrés. 


\section{Análisis comparativos por tipo de alianza familiar}

Con fines comparativos se analizó la variación de los promedios de las variables de la salud mental de los padres por tipo de alianza familiar (cooperativa, conflictiva, desorganizada) a través del test de ANOVA de un factor. Si bien se observa una tendencia a que en familias con alianzas cooperativas ambos padres presenten menores niveles de sintomatología depresiva y estrés parental, solo se observó un efecto estadísticamente significativo en los niveles de sintomatología depresiva paterna a un nivel de confianza de 95\% para los tres tipos de alianza $[\mathrm{F}(2,45)=3,3, \mathrm{p}<0,05]$.

Al repetir este análisis, pero esta vez dicotomizando los tipos de alianza en adecuadas (cooperativas) e inadecuadas (conflictiva y desorganizada), se observa la misma tendencia donde las familias cooperativas muestran promedios más bajos en sintomatología depresiva y estrés parental, sin embargo esta diferencia tampoco resulta significativa (ver tabla $\mathrm{N}^{\circ} 3$ ). No obstante, destaca nuevamente la diferencia en los niveles de sintomatología depresiva en el padre $(\mathrm{t}=1,72, \mathrm{p}=$ .091), casi alcanzando un nivel de significancia estadístico y un mediano tamaño efecto.

Adicionalmente se analizó la presencia o ausencia de sintomatología depresiva en madres y padres según el tipo de alianza familiar. De acuerdo a lo planteado por Beck, Steer y Garbin (1988) la sintomatología mínima refiere a sintomatología subclínica, por lo que los puntajes que indicaban sintomatología mínima se consideraron como ausencia de sintomatología y los puntajes que indicaban sintomatología leve, moderada o severa se consideraron como presencia de sintomatología depresiva. Los resultados reflejan una completa ausencia de sintomatología en madres y padres con alianzas cooperativas, mientras que en las alianzas conflictivas se observa el mayor porcentaje de sintomatología depresiva tanto en madres (18\%) como en padres $(20 \%)$.

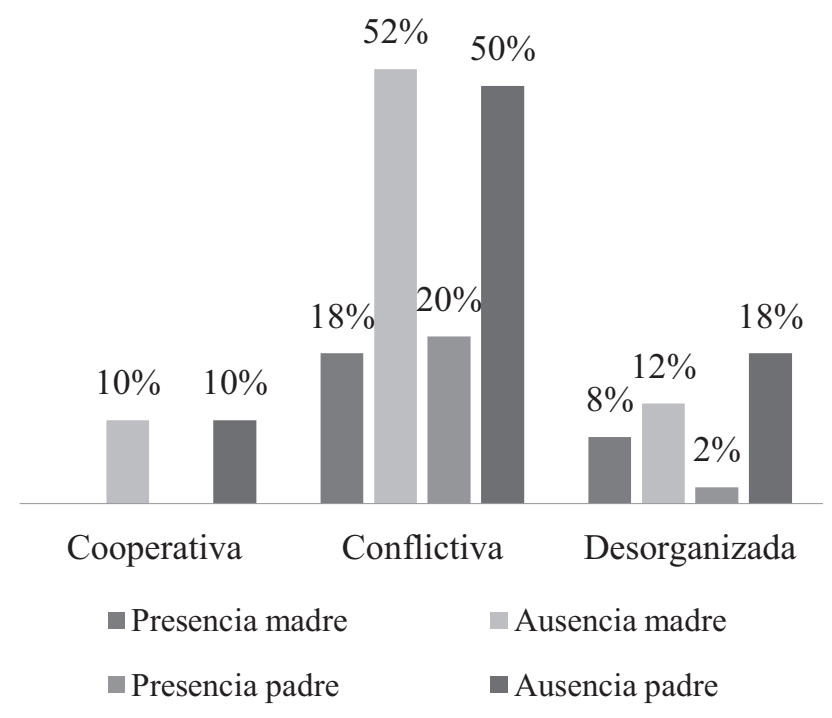

Figura 2. Presencia o ausencia de sintomatología depresiva en madres y padres según tipo de alianza familiar.

Al analizar a la "pareja parental" se formaron cuatro grupos de acuerdo a la ausencia o presencia de sintomatología: (A) ausencia de sintomatología depresiva en ambos

Tabla 2. Promedios y desviaciones de madres y padres obtenidos en el BDI y PSI-SF

\begin{tabular}{lcccc}
\hline Escala & $\begin{array}{c}\text { Media y D.S } \\
\text { Madres }\end{array}$ & $\begin{array}{c}\text { Media y D.S } \\
\text { Padres }\end{array}$ & $\begin{array}{c}\text { Prueba T } \\
\text { gl p }\end{array}$ & $\begin{array}{c}\text { Cohen } \\
\mathrm{d}\end{array}$ \\
\hline BDI & $6.85(6.09)$ & $5.42(3.94)$ & $1,6470.12$ & 0.28 \\
PSI-SF & $80.82(17.56)$ & $76.51(14.04)$ & 1.6480 .11 & 0.27 \\
PSI-SF (EP) & $30.06(8.04)$ & $27.00(6.56)$ & 2.5490 .02 & 0.42 \\
PSI-SF (IDPF) & $21.3(7.36)$ & $20.43(5.44)$ & 0.7480 .5 & 0.13 \\
PSI-SF (ND) & $29.47(8.43)$ & $28.90(6.30)$ & 0.5480 .6 & 0.08 \\
\hline
\end{tabular}

Tabla 3. Promedios y desviaciones estándar obtenidos por las madres y los padres en sintomatología depresiva y estrés parental, según el tipo de alianza familiar

\begin{tabular}{lcccc}
\hline Escala/ Tipo de Alianza & $\begin{array}{c}\text { Alianza Adecuada } \\
(\mathrm{N}=5)\end{array}$ & $\begin{array}{c}\text { Alianza Inadecuada } \\
(\mathrm{N}=45)\end{array}$ & $\begin{array}{c}\text { Prueba } \mathrm{T} \\
\operatorname{tgl~p}\end{array}$ & $\begin{array}{c}\text { Cohen } \\
\mathrm{d}\end{array}$ \\
\hline BDI Madre & $5.6(1.14)$ & $7.29(6.61)$ & 0.57480 .58 & 0.16 \\
BDI Padre & $2.6(1.52)$ & $5.74(4.01)$ & 1.72460 .09 & 0.51 \\
\hline PSI Madre & $80.2(13.08)$ & $80.53(18.07)$ & .04480 .97 & 0.01 \\
\hline PSI Padre & $68.0(10.8)$ & $77.2(14.08)$ & 1.41480 .17 & 0.41 \\
\hline
\end{tabular}


padres $(\mathrm{N}=32)$, (B) presencia de sintomatología depresiva en el padre y ausencia en la madre $(\mathrm{N}=5),(\mathrm{C})$ presencia de sintomatología depresiva en la madre y ausencia en el padre ( $N=7$ ), (D) presencia de sintomatología depresiva en ambos padres ( $\mathrm{N}=6)$. En la figura $\mathrm{N}^{\circ} 3$ se puede apreciar la distribución de estos grupos de acuerdo al tipo de alianza familiar. Destaca que en familias con alianzas cooperativas se observa una total ausencia de sintomatología depresiva en la pareja parental, sin embargo, también se observa ausencia de sintomatología en ambos padres en otros tipos de alianza, es decir, pertenecer al grupo A no es una condición excluyente de la alianza cooperativa.

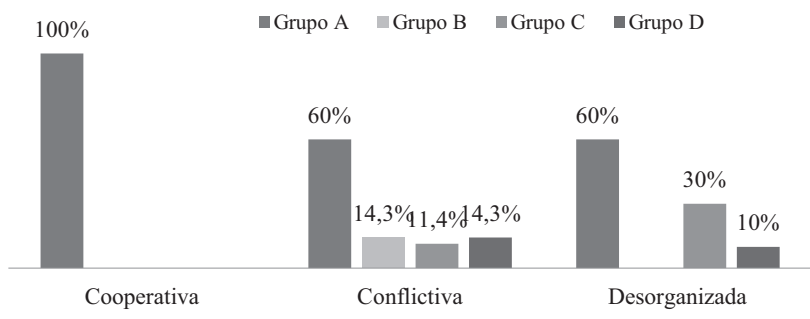

Figura 3. Presencia o ausencia de sintomatología depresiva en la pareja según tipo de alianza familiar.

En relación con el estrés parental se formaron los siguientes grupos: (A) estrés moderado en ambos padres $(\mathrm{N}=21)$, (B) estrés moderado en la madre y estrés severo en el padre $(\mathrm{N}=8),(\mathrm{C})$ estrés moderado en el padre y estrés severo en la madre ( $\mathrm{N}=14)$, (D) estrés severo en ambos padres $(\mathrm{N}=7)$. Los resultados muestran que todas las parejas donde ambos padres presentan altos niveles de estrés (Grupo D) presentan alianzas conflictivas.

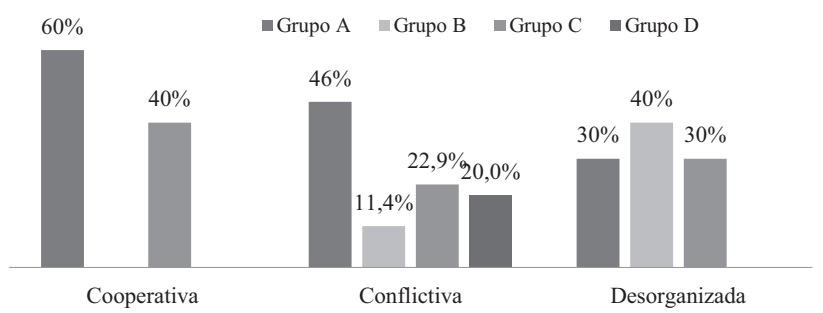

Figura 4. Estrés parental en la pareja según tipo de alianza familiar.Análisis correlacionales

Con el objetivo de conocer la relación entre la alianza familiar y las variables de salud mental de ambos padres se llevaron a cabo análisis correlacionales de tipo bicerial, pero no se encontraron correlaciones significativas a nivel estadístico.

Además se llevaron a cabo correlaciones de Pearson para analizar la relación entre las variables de salud mental de los padres. Si bien se encontraron correlaciones significativas entre la sintomatología depresiva materna y paterna $(\mathrm{r}=0,29$, $\mathrm{p}<0,05)$, entre la sintomatología depresiva materna y el estrés parental paterno $(\mathrm{r}=0,37, \mathrm{p}<0,01)$, así como entre el estrés parental de madres y padres $(r=0,33, p<0,05)$, estas correlaciones son bajas.

En relación con la salud mental parental y las características sociodemográficas, se encontró una relación negativa entre la sintomatología depresiva materna y la situación ocupacional y la jornada laboral $(\mathrm{r}=-0,34, \mathrm{p}<$ $0,05 ; \mathrm{r}=-0,32, \mathrm{p}<0,05)$ respectivamente, así como entre la escala de estrés parental y estas dos variables $(\mathrm{r}=-0,35$, $\mathrm{p}<0,05 ; \mathrm{r}=-0,29, \mathrm{p}<0,05)$. En cuanto a la sintomatología depresiva paterna se observa una correlación negativa con el nivel educacional del padre $(\mathrm{r}=-0,32, \mathrm{p}<0,05)$, así como una correlación negativa entre el estrés parental y la edad del padre $(\mathrm{r}=-0,35, \mathrm{p}<0,05)$.

\section{Discusiones}

En el presente artículo se estudiaron las relaciones entre la alianza familiar y la sintomatología depresiva y estrés en ambos padres, partiendo de la base que la salud mental tiene un impacto en la calidad de las interacciones.

Los resultados encontrados muestran que la mayoría de las familias presentaron alianzas familiares de tipo conflictiva, lo que daría cuenta la existencia de tensión en la unidad parental, la cual afectaría directamente la interacción familiar. Se evidenció competencia entre los miembros, dificultades en la coordinación, interferencias, quiebres, dificultades de reparación, así como dificultad en el logro del juego tríadico. A pesar de la tensión evidente, los padres muestran una afectividad "pseudo" positiva, lo que se observaría, por ejemplo, en la discordancia entre que los gestos faciales (sonrisa) y el tono de voz utilizado (crítica). Es importante relacionar este resultado con las características de las familias estudiadas. Se trata de familias con un grado de vulnerabilidad psicosocial, con bajo nivel educacional y con alto estrés parental. Es posible que el estrés psicosocial afecte de manera importante las dinámicas al interior de la familia, lo que se refleja en alianzas conflictivas.

Por su parte, si bien la mayoría de las madres y padres presentaron niveles mínimos de sintomatología depresiva, lo que podría esperarse dado que la muestra no constituye una muestra clínica, no ocurre lo mismo con los niveles de estrés parental, donde el total de la muestra presenta niveles moderados o severos de estrés. Las madres presentaron significativamente mayores niveles de estrés parental que los padres, lo que es coherente, dado que el $80 \%$ de las 
madres se define como cuidadora principal. Se ha descrito en estudios (McLennan y Offord, 2001) que la etapa de crianza de niños(as) pequeños es una etapa de la vida en que se presentan altos niveles de estrés debido a las exigencias afectivas, sociales y económicas del rol materno y paterno.

En relación con las hipótesis planteadas, los resultados encontrados no son concluyentes. Si bien se observa una leve tendencia a que las familias con alianzas disfuncionales presenten niveles de sintomatología depresiva y estrés parental más altos, no se encuentran diferencias estadísticamente significativas con las alianzas funcionales. Ni la presencia de sintomatología depresiva ni el estrés parental en alguno de los miembros de la pareja parental son una condición necesaria para que exista una alianza familiar disfuncional. Ahora bien, cabe destacar que la presencia de sintomatología depresiva y estrés en el padre, resulta más discriminante, especialmente entre alianzas cooperativas y conflictivas. Estos hallazgos permiten hipotetizar que hay otras variables relevantes que influyen en la alianza familiar, además de la salud mental de los padres. Es posible que si se replicara este estudio incluyendo variables de los padres (apego por ejemplo), variables de la pareja (coparentalidad, satisfacción), y variables del niño(a) (temperamento) se podría establecer un modelo comprensivo para establecer relaciones de causalidad entre las variables y la alianza familiar.

Al considerar como unidad de análisis a la pareja parental se aprecia que en las familias con alianzas funcionales ambos miembros presentan sintomatología depresiva subclínica, pero nuevamente esta condición no es excluyente de las familias con este tipo de alianzas. Cabe destacar también que todas aquellas parejas donde ambos padres presentan altos niveles de estrés se observan alianzas conflictivas. En general, aquellas familias en las cuales el padre presenta mayores índices de sintomatología depresiva y estrés parental, tienen mayor probabilidad de presentar una disfuncionalidad en la alianza familiar.

Al considerar la relación entre variables sociodemográficas y salud mental, encontramos que el tener una ocupación en las madres y mayores niveles educacionales en los padres serían factores protectores. Se podría hipotetizar que la participación en la fuerza laboral para las madres es protectora, porque constituye una instancia que amplía el rol de madre y dueña de casa, permitiéndole estar en contacto con el mundo externo, ampliar su red y mejorar su situación financiera, entre otros. Para los padres, mayores niveles educacionales resultan protectores, ya que probablemente se asocian a mayores recursos cognitivos para enfrentar distintas situaciones y conflictos, así como a mejores oportunidades laborales. Además, destaca el rol protector de la edad en los padres, lo que haría pensar que padres con mayor experiencia se encuentran más tranquilos en su rol.

Considerando que las familias evaluadas se encuentran en plena etapa de crianza y pertenecen a la población general, los hallazgos del presente estudio permiten concluir que resulta relevante el diseño e implementación de programas de prevención en salud mental, dado que la mayoría de las familias estudiadas presentan disfuncionalidad en sus interacciones familiares, así como altos niveles de estrés parental. Asimismo, resulta relevante continuar indagando otros factores que intervengan en la calidad de la alianza familiar, tanto intra como extrafamiliares.

Cabe destacar también que si bien el Lausanne Trialogue Play ha sido utilizado principalmente en investigación, constituye una herramienta clínica que permite aproximarse al diagnóstico y a la planificación de la intervención familiar, tanto desde un punto de vista estructural como dinámico. El tipo de alianza nos indica por un lado la necesidad o no de intervención psicoterapéutica para la familia, generando focos temáticos en los cuales se puede trabajar, y también permite realizar intervenciones preventivas en salud mental con niños(as) pequeños.

Dado que la calidad y diversidad de las relaciones son fundamentales en la formación del sí mismo, la unidad familiar tríadica ofrece un marco más amplio de relaciones y su evaluación permite abordar más dimensiones del contexto familiar, el desarrollo y la salud mental infantil que las permitidas por las evaluaciones díadicas. El LTP favorece la evaluación de las interacciones familiares desde múltiples perspectivas y desde distintos niveles, tanto desde la evaluación de la alianza familiar, como de posibles desajustes en la interacción que pueden tener consecuencias en el desarrollo psicológico sus miembros. Junto con esto, el LTP fomenta la incorporación del padre al contexto terapéutico, posibilitando la reflexión de la interacción familiar desde distintas perspectivas.

$\mathrm{Al}$ incorporar al padre en el abordaje de la infancia temprana se abre un campo de investigación y futura intervención que considere el sistema familiar en su globalidad y complejidad. La presencia del padre constituye un campo emergente en la psicología de niños(as) pequeños, bebés y preescolares, ya que como se señaló anteriormente, la mayoría de las investigaciones se centran en la díada madre-hijo(a). Es un desafío futuro responder a la pregunta de cómo influyen las características paternas en el vínculo con los hijos(as) y la pareja y de qué modo estas variables afectan la alianza familiar. Además, es fundamental incorporar características del niño(a) en posteriores estudios, de manera de contar con antecedentes de todos los integrantes de la tríada. 
Respecto a las limitaciones del estudio, es importante tener en cuenta que esta investigación constituye un primer acercamiento al estudio de la tríada con familias con niños(as) en edad preescolar en el contexto nacional. Sin duda que resultan necesarios más y nuevas perspectivas que amplíen y complementen los resultados aquí expuestos.

Por otro lado, el uso del BDI-I para evaluar sintomatología depresiva en población general puede haber interferido con los resultados presentados, dado que el instrumento no es preciso en discernir sintomatología en poblaciones no clínicas. Esto puede haber impactado en que la sintomatología depresiva se distribuyera de forma homogénea en la población, lo que limitó los análisis comparativos.

Asimismo, cabe destacar, que solo se realizaron análisis cualitativos con el LTP, dado que se analizó la alianza familiar. Es importante mencionar que el instrumento entrega un puntaje familiar, que es la suma de diferentes indicadores tanto estructurales como dinámicos, que enriquecen el análisis de la interacción familiar. Futuras investigaciones debiesen incoporar esta metodología.

Por último, cabe señalar que se necesitan estudios adicionales poder obtener resultados más concluyentes $\mathrm{Se}$ sugiere incorporar una evaluación de salud mental de los hijos(as), de manera de conocer de forma más directa la relación entre la alianza familiar y el estado de salud mental de todos sus integrantes. Asimismo resultaría interesante estudiar las interacciones familiares en distintos grupos sociales y clínicos, como también incoporar otras variables psicosociales que pudiesen formar parte de un modelo comprensivo más general.

\section{Referencias}

Abidin, R. R. (1995). Parenting Stress Index: Professional Manual (3rd ed.). Odessa, FL: Psychological Assessment Resources, Inc.

Beck, A.T., Ward, C.H. Mendelson, M. Mock, J. y Erlbaugh, J. (1961). An inventory for measuring depression. Archives of General Psychiatry 4, 561-71. DOI:10.1001/archpsyc.1961.01710120031004

Belsky, J. (1981). Early human experience: A family perspective. Developmental Psychology, 17, 3-23. DOI: 10.1037/0012-1649.17.1.3

Belsky, J. (1984). The determinants of parenting: A process model. Child Development, 55, 83-96. Retrieved from: http://www.jstor.org/ stable/1129836

Bronfennbrenner, U. (1987). La Ecología del Desarrollo Humano, Barcelona, Paidos.

Bronte-Tinkew, J., Moore, K.A., Matthews, G., Carrano, J., Symptoms of Major Depression in a Sample of Fathers of Infants Sociodemographic Correlates and Links to Father Involvement. Journal of Family Issues, 28, 61-99. DOI: 10.1177/0192513X06293609

Cabrera, N., Tamis LeMonda, C. S., Bradley, R. H., Hofferth, S., \& Lamb, M. E. (2000). Fatherhood in the twenty first century. Child development, 71(1), 127-136.
Centre d'Etude de la Famille (2007). Figure 2: Views according to the 4 parts of the LTP. Indications for the LTP Setting for Toddlers. VB/ 15.02 .98 ; translation : CLS y FF/2007

Clarke-Stewart, K. (1978). And daddy makes three: The father's impact on mother and young child. Child Development, 49, 466-478. Disponible en: http://www.jstor.org/journals/00093920.html.

Cowan, P. A., y Cowan, C. P. (2002). Interventions of family systems theories: Marital and family relationships in children's development and psychopathology. Development and psychopathology, 14, 731-759. DOI: $10.1017 / S 0954579402004054$

Cox, M. J., y Paley, B. (1997). Families as systems. Annual Review of Psychology, 48, 243-267. DOI: 10.1146/annurev.psych.48.1.243

Cummings, E.M., Davies, P., y Campbell, S.B. (2000). Developmental psychopathology and family process: Theory, research and clinical implications. Ney York, NY, Guilford.

Cummings, E.M., Goecke-Morey, M.C., y Raymond, J.L, (2004). Fathers in family context: Effects of marital quality and marital conflict. In M.E. Lamb (Ed.). The role of the father in child development (4th ed., pp. 222-271). New York, NY: John Wiley y Sons.

Dickstein, S., Seifer, R., Keitner, G., Miller, I., Matzko, M., Sameroff, A., et al. (1998). Levels of family assessment II: Impact of maternal psychopathology on family functioning. Journal of Family Psychology, 12, 23-40. DOI: 10.1037/0893-3200.12.1.23

Dunn J. (1991). Young children's understanding of other people: evidence from observations within the family. In Children's Theories of Mind, Frye D, Moore C (eds). Erlbaum: Hillsdale NJ.

Farkas, Ch. (2011). Informe de Confiabilidad de Instrumentos (Informe de Avance Proyecto Fondecyt $\left.N^{\circ} 1100721\right)$. Santiago, Chile: Pontificia Universidad Católica de Chile.

Favez, N., Lavanchy Scaiola, C., Tissot, H., Darwiche, J., Frascarolo, F. (2011). The Family Alliance Assessment Scales: Steps Toward Validity and Reliability of an Observational Assessment Tool for Early Family Interactions. Journal of Child and Family Studies, 20, 23-37. DOI 10.1007/s10826-010-9374-7

Feldman, R. (2007). Maternal versus child's risk and the development of parent-infant and family relationships in five high-risk populations. Development and Psychopathology, 19, 293-312. DOI: 10.1017/ S0954579407070150

Fincham, F. D. (1998). Child development and marital relations. Child Development, 69, 543-574. DOI: 10.1111/j.1467-8624.1998.tb06207.x

Fivaz $\square$ Depeursinge, E., Frascarolo, F., \& Corboz $\square$ Warnery, A. (1996). Assessing the triadic alliance between fathers, mothers, and infants at play. New Directions for Child and Adolescent Development, 74, 27-44.

Fivaz-Depeursinge, E., y Corboz-Warnery, A. (1999). The primary triangle. New York, NY: Penguin Books.

Fivaz-Depeursinge, E, Corboz-Warnery A, Keren M. (2004). The primary triangle: treating infants in their families. In Treating parent-infant relationship problems, Sameroff A, McDonough S, Rosenblum K. (eds). Guilford: New York.

Fivaz-Depeursinge, E., \& Favez, N. (2006). Exploring triangulation in infancy: Two contrasted cases. Family Process, 45(1), 3-18.

Flykt, M., Kanninen, K., Sinkkonen J. y Punamaki, R-J. (2010). Maternal Depression and Dyadic Interaction: The role of Maternal Attachment Style. Infant and Child Development, 19, 530-550. DOI: 10.1002/ icd. 679

Frascarolo, F., Favez, N., Carneiro, C., Fivaz-Depeursinge, E. (2004). Hierarchy of Interactive Functions in Father-Mother-Baby Three-way Games. Infant and Child Development,13, 301-322. DOI: 10.1002/ icd.361

Gere, M., Hagen, C., Villabo, M., Arnberg, K., Neumer, S.P. y Torgensen, S. (2013). Fathers 'Mental Health as a protective factor in the relationship between maternal and child depressive symptoms. Depression and Anxiety, 30, 31-38. DOI: 10.1002/da.22010

Gertsch Bettens, C., Favez, N., Corboz-Warnery, A. y Fivaz-Depeursinge, E. (1992). Les débuts de la communication à trois: interactions visuelles triadiques père-mère-bébé. Enfance, 46 (4), 323-348 
Gómez, E., Muñoz, M.M., Haz, A.M. (2007). Familias Multiproblemáticas y en Riesgo Social: Características e Intervención. Psykhe, 16, 43-54. DOI: $10.4067 / \mathrm{S} 0718-22282007000200004$

Hossain, Z. y Roopnarine, J. L. (1994). African-American fathers' involvement with infants: Relationship to their funcitioning style, support, education, and income. Infant Behavior and Development, 17, 175-184. DOI: 10.1016/0163-6383(94)90053-1

Koller, R. (2002). Les affects de l'enfant de trois mois dans l'interaction avec ses deux parents [The affects of the 3-month old infant during interaction with both parents]. Unpublished manuscript. Switzerland: University of Lausanne, Center for Family Studies.

Lamb, M. E. (Ed.). (2004). The role of the father in child development. John Wiley \& Sons.

Lavanchy, C. (2002). L'interaction visuelle de l'enfant de trois mois [Gaze interaction of the three-month old infant]. Unpublished manuscript. University of Lausanne, Center for Family Studies.

McHale, J. P., y Cowan, P. (1996). Understanding how family-level dynamics affect children's development: Studies of two-parent families. New Directions for Child Development. San Francisco, CA, US: Jossey-Bass.

McLanahan, S. (2009). Fragile families and the reproduction of poverty. Annals of the American Academy of Political and Social Science, 621(1), 111-131. DOI: 10.1177/0002716208324862

McLennan, J., Offord, D. (2001). Should postpartum Depression Be Targeted to Improve Child Mental Health. Journal of the American Academy of Child y Adolescent Psychiatry, 41, 28-35. doi:10.1097/00004583200201000-00008

Melipillán, R., Cova, F., Rincón, P. y Valdivia, M. (2008). Propiedades psicométricas del Inventario de Depresión de Beck-II en adolescentes chilenos. Terapia Psicológica, 26 (1), 59-69. DOI: 10.4067/S071848082008000100005

Ontai, L. L., \& Thompson, R. A. (2002). Patterns of attachment and maternal discourse effects on children $>$ s emotion understanding from 3 to 5 years of age. Social Development, 11(4), 433-450.

Parke, R., Coltrane, S., Duffy, S., Buriel, R., Dennis, J, Powers, J, et al., (2004). Economic stress, parenting, and child adjustment in Mexican American and European families. Child Development, 75, 1613-1631.

Pérez, J., Lorence, B., Menéndez, S. (2010). Estrés y competencia parental: un estudio con madres y padres trabajadores. Suma Psicológica, 17, 47-57. Disponible en: http://redalyc.uaemex.mx/src/inicio/ArtPdfRed. jsp?iCve=134215244004

Phillip, D., Fivaz-Depeursinge, E., Corboz-Warnery, A., y Favez, N. (2009). Young infants'triangular communication with their parents in the context of maternal postpartum psychosis: four case studies. Infant Mental Health Journal, 30(4), 341-365. DOI: 10.1002/imhj.20218

Pleck, J., y Masciadrelli, B.P. (2004). Paternal involvement in U.S. residential fathers: Levels, sources, and consequences In ME. Lamb (ed.), The role of the father in child development (4th ed., pp. 222-271). New York, NY: John Wiley y Sons.

Rodrigo; M.J., Martín, J.C., Cabrera, E., Máiquez, M.L. (2009): Las Competencias Parentales en Contextos de Riesgo Psicosocial. Intervención Psicosocial, 18, 113-120. Disponible en: < http://scielo.isciii.es/scielo. php?script $=$ sci_arttextypid=S1132-05592009000200003ylng=esynrm $=$ iso $>$. ISSN 1132-0559.

Roggman, L. A., Moe, S. T., Hart, A. D., y Forthun, L. F. (1994). Family Leisure an social support: Relations with parenting stress and psychological well-being in Head Start parents. Early Childhood Research Quarterly, 9, 463-480. DOI: 10.1016/0885-2006(94)90020-5

Seifer, R., y Dickstein, S. (2000). Parental mental illness and infant development. In C.H. Zeanah Jr. (Ed.), Handbook of infant mental health (2nd ed., pp. 145-160). New York: Guilford.

Stern, D. (1977). The first relationship: Infant and mother. Cambridge, MA: Harvard University Press.

Tenorio, Sh., Santelices M.P., Pérez, C. (2009) Apego, sensibilidad paterna y patrón de interacción del padre con su primer bebé. Revista Argentina de Clínica Psicológica. 68, 1, 51-58.

Teti, D.M., Gelfand, D.M., Messinger, D.S., y Isabella, R. (1995). Maternal Depression and the Quality of Early Attachment: An Examination of
Infants, Preschoolers, and Their Mothers. Developmental Psychology, 31(3), 364-376. DOI: 10.1037/0012-1649.31.3.364

Vick Whittaker, J., Jones Harde, B., See, H., Meisch, A., Westbrook, T'P. (2011). Family risks and protective factors: Pathways to Early Head Start toddlers'social-emotional functioning. Early Childhood Research Quarterly, 26, 74-86. DOI: 10.1016/j.ecresq.2010.04.007

Weinberg, M. K., Tronick, E. Z. (1998) The impact of maternal psychiatric illness on infant development. Journal of Clinical Psychiatry, 59, 53-61. DOI: 10.1542/peds.102.5.SE1.1298

Yogman, M. (1981). Games fathers and mothers play with their infants. Infant Mental Health Journal, 2(4), 241-248. DOI: $10.1002 / 1097-0355(198124) 2: 4<241:: A I D$ IMHJ2280020406>3.0.CO;2-8 
